\title{
Macrophage Migration Inhibitory Factor deficiency attenuates kidney Injury by downregulating cytokines via CYR61 in lupus-prone mice induced by pristane.
}

\section{Yang Tu}

Shanghai Jiao Tong University School of Medicine https://orcid.org/0000-0003-0142-561X

\section{Baoxiang Wang}

Department of neurology, the first Hospital of JiaXing

\section{Suli Wang}

Shanghai Jiao Tong University School of Medicine Affiliated Renji Hospital

\section{Chaofan Fan}

Shanghai Jiao Tong University School of Medicine Affiliated Renji Hospital

\section{Xinyu Meng}

Shanghai Jiao Tong University School of Medicine Affiliated Renji Hospital

\section{Lin Leng}

Yale University school of Medicine

\section{Richard Bucala}

Yale University School of Medicine

Jia Li

Shanghai Jiao Tong University School of Medicine Affiliated Renji Hospital

Liangjing Lu ( $\nabla$ lu_liangjing@163.com )

\section{Research article}

Keywords: SLE, Lupus nephritis, MIF, CYR61, macrophages

Posted Date: February 26th, 2020

DOI: https://doi.org/10.21203/rs.2.24616/v1

License: (a) (1) This work is licensed under a Creative Commons Attribution 4.0 International License. Read Full License 


\section{Abstract}

Background Lupus nephritis ( $L N)$ is one of the most serious manifestations of systemic lupus erythematous (SLE) and accounts for significant mortality and morbidity. Previous research has demonstrated that macrophage migration inhibitory factor (MIF) is involved in the pathogenesis of lupus nephritis, but the detailed mechanism is not elucidated. The aim of this study was to explore the pathogenesis of MIF in lupus nephritis with the pristane-induced mouse model of SLE.

Methods Mif-/-mice and Wild type mice in the C57BL/6 (B6) background were used to induce Lupus model by pristane. 24 hour urine and sera were collected in the sixth month and mice were sacrificed to harvest tissues. Serum ANA, anti-dsDNA antibodies, C3, urinary creatinine and albumin were detected by ELISA. Related inflammatory cytokines were detected by Bio-Plex Pro $^{\mathrm{Tm}}$ assays and ELISA. CYR61 mRNA expression was detected by RT-qPCR and CYR61 protein expression were detected by Western blot. Immunofluorescence was used to detect the expression of MIF, ICs and C3 deposition and related cytokines expression in the kidneys. Immunohistochemical staining was used to detect macrophage infiltration and periodic acid-Schiff (PAS) staining were used for kidney histology. The Mann-Whitney test and Student's t test were used to compare multiple group differences. The correlation were analyzed by Spearman correlation.

Results Mif -/- mice with pristane-induced SLE have less inflammatory cytokines expression in sera. The Mif-/- mice have reduced renal injury, less macrophage infiltration, CYR61 and inflammatory cytokines expression in the kidneys. MIF induced the expression of CYR61, which can induce the expression of IL$1 \beta$, IL- 6 and MCP-1 in bone marrow-derived macrophages (BMDM) in a cell-based assay.

Conclusions The results suggest that MIF plays an important role in kidney injury by inducing macrophages infiltration and inflammatory cytokines expression in situ. Our finding support the pathogenic contribution of high expression MIF alleles in SLE and suggest that MIF antagonism might offer an effective therapeutic option in lupus nephritis.

\section{Introduction}

Systemic lupus erythematosus (SLE) is a prototypic autoimmune disease characterized by autoantibodies directed against nuclear antigens and chronic inflammation affecting multiple organs [1, 2]. The kidney is one of the most common organs involved in SLE and lupus nephritis affects more than $60 \%$ SLE patients, leading to deleterious clinical outcomes [3,4]. Although supportive care and new therapeutic advances have improved the prognosis of SLE, both end-stage renal disease and overall mortality remain excessive $[5,6]$.

Numerous studies have revealed that the immunoregulatory cytokine MIF participates in the pathogenesis of SLE with respect to both disease initiation and progression [7]. MIF expression is increased in SLE patients and positively correlates with disease activity [8]. MIF is overexpressed in lupusprone mice and renal injury is alleviated in Mif -/- MRL/Ipr mice [9]. Furthermore, MIF antagonism can 
reduce glomerular injury by reducing the expression of inflammatory cytokines TNF- $a, I L-1 \beta$ and CCL2 in lupus-prone NZB/NZW F1 and MRL/Ipr mice [10]. Intraperitoneal injection of pristane in different strains of mice, such as C57BL/10 and BALB/c, induces lymphoproliferation and a lupus-like disease that includes glomerulonephritis $[11,12]$. To better understand the role of MIF in the pathogenesis of lupus nephritis, pristane was administered to C57BL/ 6 (wild-type) and MIF knockout (Mif-/-, C57BL/6 background) mice to examine the development of lupus nephritis models. This study revealed that MIF aggravates lupus nephritis by promoting renal macrophage accumulation and inflammatory cytokine expression.

\section{Materials And Methods}

\subsection{Mice}

MIF gene knockout mice in the C57BL/6 (B6) background (Mif-/-) were provided by Professor Donghong Cui from Shanghai Mental Health Center Shanghai Jiao Tong University School Medicine, Shanghai, China (ref this strain with Fingerle -Rowson et al PNAS 2003). Wild type C57BL/6 (B6) mice were purchased from Shanghai SLAC Laboratory Animal Co., Ltd (Shanghai, China). All mice were housed in the animal facility of Shanghai Renji Hospital and received food and water ad libitum. Female mice at 810 weeks of age were used for all experiments. Lupus mice were induced by a single intraperitoneal injection of $0.5 \mathrm{ml}$ pristane (Sigma) $[13,14]$. Twentyu-four hour urine and sera were collected in the sixth month and mice were sacrificed to harvest tissues. All animal experiments complied with the relevant institutional guidelines of animal ethics from Renji Hospital, Shanghai Jiao Tong University School of Medicine.

\subsection{Histological evaluation}

Kidneys were fixed in $4 \%$ paraformaldehyde and embedded in paraffin. Sections ( $4 \mu \mathrm{m}$ thick) were stained with periodic acid-Schiff (PAS), the ratio of glomerular PAS-positive area to the glomerular tuft area was quantified by using ImageJ (NIH), and the mesangial matrix score calculated [15].

\subsection{Immunofluorescence and histochemical staining}

The $4 \mu \mathrm{m}$ kidney sections were permeabilized in $0.05 \%$ TritonX-100 (Servicebio, Wuhan, China) for 10 min, blocked in 1\% BSA for $1 \mathrm{~h}$ at room temperature, and incubated with antibodies directed against MIF (1:100, abcam, ab227073), IgG2a (1:100, abcam, ab190463), C3 (1:100, abcam, ab97462), IL-1ß (1:100, abcam, ab2015), IL-6 (1:100, abcam, ab6672) and MCP-1 (1:100, Abcam, ab9899) at $4^{\circ} \mathrm{C}$ overnight with protection from light. On the second day, the sections were incubated with IgG2a and C3, and washed and incubated with secondary rabbit IgG antibodies conjugated with Alexa Fluor ${ }^{\circledR} 488(1: 100$, Abcam, ab150077) for $1 \mathrm{~h}$ with protection from light. Fluorescence microscopy (Nikon Eclipse C1; Nikon Corporation) was used to acquire micrographs. Immunohistochemical staining was employed to detect macrophage infiltration, and the kidney sections were deparaffinized by Xylene and pretreated with different concentration ethanol (varies from $100 \%$ to $70 \%$ ), followed by antigen retrieval with $0.01 \mathrm{M}$ 
citrate buffer. Background staining was blocked with $1 \%$ BSA, and sections then were incubated with antiMac-2 (1:100, Abcam, ab76245) in $4{ }^{\circ} \mathrm{C}$ overnight, followed by secondary antibody, the ABC reagent and diaminobenzidine chromogen. Image $\mathrm{J}$ software were used to quantify Mac-2 staining areas and the mean fluorescence of MIF, IgG, C3, IL-1 $\beta$, IL- 6 and MCP-1. More than 20 glomeruli were measured for each sample.

\subsection{Bio-Plex Pro ${ }^{\mathrm{m}}$ assays}

Inflammatory cytokines, including IL-1a, IL-1 $\beta$, IL-6, MCP-1, TNF-a, IL-2, IL-4, IL-5, IL-10, IL-12 (p40), IL-12 (p70), IL-13, IL-17A, G-CSF, GM-CSF, IFN-g, MIP-1 and MIP-1 were detected by the magnetic bead-based multiplex Bio-Plex Pro ${ }^{\mathrm{TM}}$ assay kit (Bio-Rad Laboratories) according to the manufacturer's instructions. The Bio-Plex 200 system and the Bio-Plex Manager software (Bio-Rad Laboratories) were used to analyze the results.

\subsection{BMDM cells culture, transfection and stimulation}

High-glucose DMEM medium was supplemented with 20\% L929 cell supernatants and $10 \%$ Fetal Bovine Serum (FBS) to generate L929-Conditioned Medium. Bone marrow cells were iolated from MIF-/- and C57BL/ 6 wild type mice, treated with RBC lysis buffer, enumerated, and cultured in $2 \times 10^{6}$ cells in 6 -well plates with $2 \mathrm{~mL}$ L929-Conditioned Medium to generate bone marrow-derived macrophages (BMDM) Two $\mathrm{ml}$ of fresh medium was added very 3 days. On day 7, the BMDM were transfected with CYR61 siRNA (siCYR61, purchased from Genepharma, Shanghai) with Lipofectamine RNAiMAX (Invitrogen), or transfected with pCMV6-CYR61 vector (CYR61, purchased from Origene, cat. Number: MC208761) with Lipo3000 (Invitrogen) according to the manufacturer's instructions. After the BMDM were transfected with siCYR61 or CYR61 vector for $24 \mathrm{hrs,} \mathrm{MIF}(100 \mathrm{ng} / \mathrm{ml})$ were added to stimulate the BMDM for $6 \mathrm{hrs}$; the cells and culture supernatants then were collected to analyze CYR61and inflammatory cytokines expression.

\subsection{Enzyme-linked immunosorbent assay (ELISA)}

Protein expression levels of IL-1 $\beta$, IL- 6 and MCP- 1 in mice sera, and IL-1 $\beta$, IL- 6 and MCP- 1 in BMDM cells supernatants were measured by enzyme-linked immunosorbent assay (ELISA) kit (R\&D). Urine albumin and urinary creatinine expression levels were examined with ELISA kits according to the manufacture's instruction(Sigma-Aldrich. Urine albumin creatinine ratios are presented as micrograms of urinary albumin per milligram of urinary creatinine.

\subsection{Quantitative real-time polymerase chain reaction (RT-PCR)}

TRizol reagent (Invitrogen) was used to extract total RNA and the quantities of mRNA were detected by PrimeScript RT Reagent kit (Takara) and SYBR Premix Ex Taq RT-PCR kit (Takara). Gene expression was normalized to tubulin as the internal control by using $2^{-\Delta \Delta C t}$ method.

\subsection{Western blot}


BMDM cells were harvested in lysis buffer and heated at $99^{\circ} \mathrm{C}$ for 10 mins. The lysates then were subjected to $10 \%$ sodium dodecyl sulfate-polyacrylamide gel electrophoresis (SDS-PAGE) and transferred to PVDF membranes (Millipore). The membranes were blocked with $8 \%$ skim milk for $1 \mathrm{hr}$, washed with TBST buffer, and the membranes incubated with primary antibodies directed against tubulin (purchased from Cell Signaling Technology, Catalog 5335T), CYR61 (CST, Catalog 14479T) at $4^{\circ} \mathrm{C}$ overnight. After incubation with HRP-conjugated secondary antibody (1: 2000, CST, Catalog 14708S), the membranes were washed and the bands were visualized by chemiluminescence detection kit (Thermo). Anti-tubulin antibody was used as internal control. Protein expression was quantified by density-analysis software (Image J).

\subsection{Statistical analysis}

Data obtained from three independent experiments were analyzed by Graphpad Version 7, and multiple group differences were compared using one-way ANOVA with the Mann-Whitney test or Student's t test and presented as mean \pm SD (standard deviation). Correlations were analyzed by Spearman correlation. $P$ $<0.05$ was considered significant.

\section{Results}

\subsection{The kidney injury is alleviated in Mif/- mice with pristane induced lupus}

To explore whether kidney injury is alleviated in Mif-/- mice with pristane-induced lupus, we first compared the urinary albumin/creatinine ratio between Mif-/- C57BL/6 and Mift/+ C57BL/6 (wild-type) mice, and found it to be significantly decreased in the Mif-/- pristane group $(2.408 \pm 0.332 \mathrm{mg} / \mathrm{mg}$ creatinine $)$ compared with the pristine-treated wild-type group $(4.496 \pm 0.697 \mathrm{mg} / \mathrm{mg}$ creatinine) $(P \otimes 0.01$, Fig. 1A). Kidneys were harvested and the pathology of the kidneys examined. There was clear pathological damage in kidneys consistent with nephritis in mice that received pristine, and MIF expression was significantly increased in the wild-type mice (Fig. 1B, Fig 1C). To examine whether MIF exacerbates immune complex (IC) and complement 3 (C3) deposition in glomeruli, we examined the expression of ICs ( $\mathrm{IgG})$ and C3 in the kidneys. There were less IC and C3 deposition in the kidneys from Mif-/- mice than in wild-type mice (Fig. 1B, D, E). The PAS-positive area in wild-type mice treated with pristane was significantly larger than in corresponding Mif-/- mice ( $P \otimes 0.05$, Fig. 1B, F), indicating that Mif-/- mice treated with pristane had less histological injury in the kidney. Previous studies have demonstrated that macrophage infiltration plays an important role in SLE-induced kidney injury and that intrarenal macrophage content is a prognosticator for severe glomerular damage [9]. The expression of the macrophage marker MAC-2 in glomerulus was detected in all groups and there was little macrophage infiltration detectable in mice not treated with pristine. Macrophage infiltration increased significantly after pristane treatment,and there was less macrophage infiltration in Mif-/-pristane-treated mice compared with pristane-treated wild-type mice $(P \otimes 0.01$, Fig. 1B, G). These results indicate that MIF aggravates kidney injury in C57BL/ 6 mice with pristane induced lupus model. 


\subsection{Serum autoantibodies expression is similar in Mif/- and wild type C57BL/6 mice with pristane- induced lupus.}

To explore more closely the mechanism of MIF action in lupus nephritis, we examined autoantibodies and complement $\mathrm{C} 3$ expression in sera. After pristane treatment, the expression of ANA and anti-dsDNA antibodies were significantly increased, and sera concentrations of C3 were significantly decreased. However, no significant differences were observed between the pristane-treated Mif-/- and wild-type groups (Fig. 2A, B, C). Under control (untreated) conditions, the spleen weights in Mif-/- mice also similar to that of wild-type C57BL/6 mice, however, the volume and weights of spleens in Mif-/-mice were significantly decreased after treatment with pristane ( $P \otimes 0.01$, Fig. 2D, E).

\subsection{Mif-/- mice with pristane-induced SLE have less inflammatory cytokine expression in sera when compared to wild type mice with pristane-induced SLE.}

It is known that MIF can promote the expression of multiple inflammatory cytokines. To examine whether MIF can influence cytokine expression in the pristane model of lupus, Bio-Plex Pro ${ }^{\mathrm{TM}}$ assays were used to quantify 18 cytokines, including IL-1a, IL-1 3 , IL-6, MCP-1, TNF-a, IL-2, IL-4, IL-5, IL-10, IL-12 (p40), IL-12

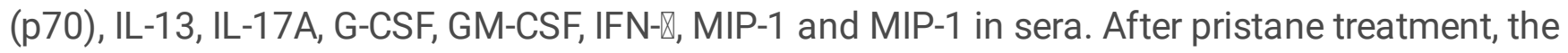
expression of IL-1 $\beta$, IL- 6 and MCP-1 in Mif-/- mice were significantly increased (Fig. 3A). To confirm the results of the Bio-Plex Pro ${ }^{\text {TM }}$ assays, the expression of IL-1 $\beta$, IL- 6 and MCP- 1 also was measured by ELISA. IL-1 $\beta$, IL- 6 and MCP-1 were significantly increased in the sera from wild type C57BL/ 6 mice when compared with Mif-/-mice ( $P \otimes 0.01$, Fig. 3B, C, D). To assess whether the relative decrease in inflammatory cytokine expression was associated with reduced renal injury, we examined the relationship between cytokine expression in sera and PAS-positive areas in kidneys. We found the expression of IL-1 $\beta$, IL-6, and MCP-1 to be positively associated with the PAS-positive areas in kidney (P凶0.05, Fig. 3E, F, G). These results indicate that decreased systemic inflammatory cytokines expression contribute to the reduction in kidney injury observed in lupus mice.

\subsection{MIF promotes the expression of inflammatory cytokines IL-1 $\beta$, IL-6 and MCP-1 in pristane-induced SLE.}

We next examined the expression of IL-1 $1 \beta$, IL- 6 and MCP- 1 in the renal glomeruli and quantitated the fluorescence intensity by image $\mathrm{J}$. We found that there were less IL-1 $\beta$, IL- 6 and MCP-1 expression in the glomerulus in Mif-/- mice when compared to wild-type mice (Fig. 4A, B, C, D).

\subsection{MIF promotes the expression of IL-1 $\beta$, IL-6 and MCP-1 and CYR61 in BMDMs.}

To further verify that MIF can promote inflammatory cytokines expression in macrophages in experimentally-induced SLE, we generated BMDM cells both from wild-type and Mif-/- mice, and stimulated them with recombinant MIF. The expression of IL-1 $\beta$, IL- 6 and MCP- 1 by BMDMs from Mif-/mice was significantly decreased when compared with wild type mice (Pख0.001, Fig. 5A, B, C), and MIF treatment significantly increased the expression of IL-1 $\beta$, IL-6 and MCP-1 (Pख0.001, Fig. 5A, B, C). These 
results showed that MIF promotes the expression of IL-1 $\beta$, IL-6 and MCP-1 in macrophages, which participate in the pathogenesis of kidney injury.

To explore potential mechanisms by which MIF increases the expression of inflammatory cytokines, we analyzed a GEO datasets (GSE10241) for the whole-genome expression in MIF knockdown HEK293 cells and the control HEK293 cells. Among the 15 genes identified to show the greatest reduction in expression in MIF gene deficient cells (Fig. 5D), 3 genes: CYR61, ANKRD1 and CTGF, are key molecules involved in the Hippo signaling pathway. Emerging evidence has demonstrated that the Hippo signaling pathway is a key regulator in organ size control, tissue regeneration, tumor suppression and the immune system in multiple organisms $[16,17]$. We therefore examined the expression of CYR61, ANKRD1 and CTGF in the kidneys, and found that there was reduced CYR61 expression in Mif-/- mice (Pख0.001, Fig. 5E), and no significant differences in the expression of ANKRD1 and CTGF (data were not shown). We also examined the CYR61 mRNA and protein expression in BMDM, and we found that both the CYR61 mRNA and protein expression in BMDM were significantly reduced in BMDM cells (Pख0.01, Fig. 5F, G). MIF treatment also significantly increased the expression of CYR61 mRNA and protein in vitro (P凶0.05, Fig. 5F, G).

\subsection{MIF induces the expression of IL-1 $\beta$, IL-6 and MCP-1 via CYR61}

Recent studies have supported a critical role for Hippo signaling in autoimmunity [18], and that CYR61 blockade attenuates renal inflammation [19]. We examined the hypothesis that MIF may induce the expression of IL-1 $\beta$, IL- 6 and MCP-1 via CYR61 by overexpressing CYR61 in WT and Mif-/- BMDM cells. The increased expression of CYR61 was evident under our experimental conditions ( $P \otimes 0.01$, Fig. 6A), and was associated with a coincident increase in the expression of IL-1 $\beta, I L-6$ and MCP-1 (P凶0.01, Fig. 6B, C, D). We also knocked down CYR61 expression by CYR61 siRNA (P凶0.001, Fig. 6E) and observed a significant reduction in the expression of IL-1 $\beta$, IL-6 and MCP-1 (P凶0.05, Fig. 6F, G, H).

\section{Discussion}

The biological activity of MIF as a soluble immunologic mediator was first described in 1966 [20]. After the definitive cloning of MIF in 1993 [21], studies using recombinant MIF, neutralizing antibodies and MIFdeficient mice demonstrated that this mediator is a pivotal, upstream regulator of both the innate and the adaptive immune response $[22,23]$. By binding to its cognate receptor CD74 and its associated noncognate CXCR2 and CXCR4 receptors, MIF is a major regulator of inflammatory cell recruitment [24]. By activating the NLRP3 inflammasome, MIF promotes the expression and secretion of IL-1 $\beta$ [25]. MIF additionally is involved in the differentiation of the adaptive $T$ and $B$ cell response, and it can also influence the survival and activation of lymphocytes $[23,26,27]$.

Clinical studies have shown that the expression level of MIF is elevated in SLE patients and is positively associated with disease activity $[8,28,29]$. Renal MIF expression is markedly up-regulated in patients with proliferative glomerulonephritis and correlates with leukocyte infiltration, glomerular injury, and renal function impairment [30]. Genetic studies also have identified that functional MIF promoter polymorphisms are associated with SLE susceptibility, clinical manifestations, and outcome [31, 32, 33]. 
MIF expression is elevated in lupus-prone mice, and renal injury is alleviated in Mif-/- MRL/Ipr mice, with reduced renal macrophage recruitment and glomerular IgG deposition [9]. Both anti-MIF antibody and the pharmacologic MIF antagonist ISO-1 alleviates glomerulonephritis by inhibiting CD74 + leukocyte recruitment into kidneys, and reducing inflammatory cytokine and chemokine expression in the two lupusprone NZB/NZW F1 and MRL/Ipr mouse strains. Notably, the circulating levels of autoantibodies against double-stranded DNA were not affected in the two lupus-prone strains treated with ISO-1 MIF or anti-MIF antibody, pointing to a predominant action of MIF on innate pathways of tissue damage [10].

Circulating antibodies deposit in glomeruli and contribute to the formation of immune complexes, which in turn aggravate renal injury [34]. Both IC deposition in glomeruli and circulating autoantibody concentrations can reflect the renal injury, and there is data to suggest that MIF may influence on IgG deposition in the kidney [9]. In the present study, we found that MIF is upregulated in the glomerulus in mice wth pristane-induced lupus, and that renal inflammation and injury is alleviated with reduced IgG and C3 deposition in the glomeruli in the setting of genetic Mif deficiency. Additionally, there was reduced macrophage infiltration in the glomeruli of Mif-/- mice. Notably, serum ANA, anti-dsDNA antibodies and C3 concentrations were similar in the two groups of lupus mice induced by pristine, which is consistent with previous studies indicating no major alterations in autoantibody production but a reduction in immune complex deposition in the glomeruli in -/- MRL/Ipr mice [9]. It is known that podocyte integrity plays a role in glomerular permeability. In a MIF-transgenic mouse model, it was observed that MIF overexpression induces podocyte injury, resulting in proteinuria and progressive renal failure [35].

The inflammatory cytokines IL-1 $1 \beta$, IL- 6 and MCP-1 are critical nephritogenic cytokines. IL-1 $\beta$ can accelerate disease progression in lupus-prone $\mathrm{MRL} / \mathrm{Ipr}$ mice $[36,37]$ and studies suggest that serum IL-1 $\beta$ expression is associated with disease activity in SLE patients [38, 39]. IL-6 is produced both by leukocytes and intrinsic kidney cells, and can promote mesangial cell proliferation as well as stimulate B-cell differentiation and autoantibody secretion $[40,41]$. In both murine and in human SLE, IL-6 expression is increased in serum and glomeruli, and is correlated with disease activity [42]. MCP-1 can promote the transendothelial migration of monocytes, which in turn facilitates tissue destruction [43]. It is observed that urinary MCP-1 expression is elevated in Mif $-/-\mathrm{MRL} / \mathrm{Ipr}$ mice and is associated with macrophage recruitment in kidney [9]. Genetic studies also showed that a polymorphism in the MCP-1 gene is associated with nephritis in [44].

MIF promotes the expression of many downstream inflammatory cytokines. We found that the expression of IL-1 $\beta$, IL- 6 and MCP-1 both in sera and in glomeruli were significantly decreased in Mif-/- mice after treatment with pristane. We also found that the expression of inflammatory cytokines IL-1 $\beta$, IL- 6 and MCP-1 was associated with kidney injury in this lupus model.

We found MIF to upregulate the expression of CYR61 both in mouse and in BMDM cells. Previous studies have demonstrated that CYR61 can induce the expression of IL-1 $\beta$, IL- 6 and MCP-1 [19, 45, 46, 47], and it has also been reported that serum Cyr61 expression is associated with inflammatory disease activity in SLE patients [48]. The present finding indicate that MIF can upregulate the expression of CYR61 in 
BMDMs, and that MIF promotes the expression of IL-1 $\beta$, IL- 6 and MCP-1 in BMDMs. However, whether MIF can regulate inflammatory cytokines expression exclusively via CYR61 remains to be determined.

In conclusion, our data in the pristane-induced mouse model of lupus support an important role for MIF in the progression of kidney injury by inducing macrophage infiltration and promoting the expression of inflammatory cytokines via CYR61. The MIF antagonist milatuzumab, which blocks MIF signaling through its cognate receptor CD74, is currently in clinical evaluation and may be an effective therapeutic approach in human lupus nephritis,

\section{Abbreviations}

LN: Lupus nephritis; SLE: systemic lupus erythematous; MIF: macrophage migration inhibitory factor; Mif-/: MIF gene knockout; PAS: periodic acid-Schiff; RT-qPCR: real-time quantitative polymerase chain reaction; BMDM: bone marrow-derived macrophages; dsDNA: Anti-double strand DNA; C3: complement 3; SD: standard deviation.

\section{Declarations}

\section{Ethics approval and consent to participate:}

The study complied with the Declaration of Helsinki and was approved by the Ethics Committee of Renji Hospital, Shanghai, China.

\section{Consent for publication:}

Not applicable

\section{Availability of data and materials:}

The datasets used and/or analysed during the current study are available from the corresponding author on reasonable request.

\section{Authors' contributions:}

Yang Tu completed this research and wrote the paper. Jia Li and Liangjing Lu designed the research and revised the paper. Baoxiang Wang, Suli Wang and Yuetian Yu critically revised the paper. Xingyu Meng help finished the animal experiments. Jia Li and Liangjing Lu approved the article for publication.

Conflict of Interest: Richard Bucala is a co-inventor on patents describing the potential therapeutic utility of MIF antagonism. The remaining authors declare no conflict of interest.

Funding: This work was supported by grants from the National Key Research and Development Program of China (2017YFC0909002), the National Natural Science Foundation of China $(81102267,81373209)$, 
Innovation Program of Shanghai Municipal Education Commission (\#12YZ055) and the Special Research Foundation for the Doctoral Program of Higher Education (\#201100731120091).

\section{References}

1. Dörner Thomas, Furie Richard. Novel paradigms in systemic lupus erythematosus. Lancet, 2019, 393 : 2344-2358.

2. Durcan Laura, O'Dwyer Tom, Petri Michelle, Management strategies and future directions for systemic lupus erythematosus in adults. Lancet, 2019, 393: 2332-2343.

3. Bernatsky S, Boivin JF, Joseph L, et al. Mortality in systemic lupus erythematosus. Arthritis Rheum 2006; 54: 2550-7.

4. Kasitanon N, Magder LS, Petri M. Predictors of survival in systemic lupus erythematosus. Medicine (Baltimore) 2006; 85: 147-56.

5. Tektonidou MG, Lewandowski LB, Hu J, et al. Survival in adults and children with systemic lupus erythematosus: a systematic review and Bayesian meta-analysis of studies from 1950 to 2016. Ann Rheum Dis 2017; 76: 2009-16.

6. Tektonidou MG, Dasgupta A, Ward MM. Risk of end-stage renal disease in patients with lupus nephritis, 1970 to 2015 a systematic review and bayesian meta-analysis. Arthritis Rheum 2016, 68: 1432-41.

7. Kang Insoo, Bucala Richard. The immunobiology of MIF: function, genetics and prospects for precision medicine. Nat Rev Rheumatol, 2019, 15: 427-437.

8. Feng $X$, Chen W, Xiao L, et al. Artesunate inhibits type I interferon-induced production of macrophage migration inhibitory factor in patients with systemic lupus erythematosus. Lupus, 2017, 26: 62-72.

9. Hoi Alberta Y, Hickey Michael J, Hall Pamela, et al. Macrophage migration inhibitory factor deficiency attenuates macrophage recruitment, glomerulonephritis, and lethality in MRL/lpr mice. J Immunol, 2006, 177: 5687-96.

10. Leng Lin, Chen Liang, Fan Juan, et al. A small-molecule macrophage migration inhibitory factor antagonist protects against glomerulonephritis in lupus-prone NZB/NZW F1 and MRL/Ipr mice. J Immunol., 2011, 186: 527-38.

11. Satoh M, Reeves W H. Induction of lupus-associated autoantibodies in BALB/c mice by intraperitoneal injection of pristane. J Exp Med, 1994, 180: 2341-6.

12. Chowdhary V R, Grande J P, Luthra H S, et al. Characterization of haemorrhagic pulmonary capillaritis: another manifestation of Pristane-induced lupus. Rheumatology (Oxford), 2007, 46: 1405-10.

13. Gottschalk TA, Tsantikos E, Hibbs ML. Pathogenic Inflammation and Its Therapeutic Targeting in Systemic Lupus Erythematosus. Front Immunol. 2015 Oct 28;6:550.

14. Fingerle-Rowson $\mathrm{G}$, Petrenko $\mathrm{O}$, Metz $\mathrm{CN}$, et al. The p53-dependent effects of macrophage migration inhibitory factor revealed by gene targeting. Proc Natl Acad Sci U S A. 2003 Aug 5;100 (16):9354-9. 
15. Imaruoka Kenta, Oe Yuji, Fushima Tomofumi et al. Nicotinamide alleviates kidney injury and pregnancy outcomes in lupus-prone MRL/Ipr mice treated with lipopolysaccharide. Biochem. Biophys. Res Commun, 2019, 510: 587-593.

16. Hwang SM, Jin M, Shin YH, etc. Role of LPA and the Hippo pathway on apoptosis in salivary gland epithelial cells. Exp Mol Med. 2014 Dec 12;46:e125. doi: 10.1038/emm.2014.77.

17. Hong L, Li X, Zhou D, et al. Role of Hippo signaling in regulating immunity. Cellular \& Molecular Immunology. 2018 Dec;15(12):1003-1009. DOI: 10.1038/s41423-018-0007-1.

18. Zhang Y, Zhang H, Zhao B. Hippo Signaling in the Immune System. Trends in Biochemical Sciences, 2017.

19. Lai CF, Lin SL, Chiang WC, etc. Blockade of cysteine-rich protein 61 attenuates renal inflammation and fibrosis after ischemic kidney injury. Am J Physiol Renal Physiol. 2014 Sep 1;307 (5):F581-92. doi: 10.1152/ajprenal.00670.2013.

20. Bloom B R, Bennett B. Mechanism of a reaction in vitro associated with delayed-type hypersensitivity. Science, 1966, 153: 80-2.

21. Bernhagen J, Calandra T, Mitchell RA, et al. MIF is a pituitary-derived cytokine that potentiates lethal endotoxaemia. Nature. 1993 Oct 21;365 (6448):756-9.

22. Calandra T, Bernhagen J, Mitchell R A, et al. The macrophage is an important and previously unrecognized source of macrophage migration inhibitory factor.[J] .J. Exp. Med., 1994, 179: 1895902.

23. Bacher $\mathrm{M}$, Metz $\mathrm{C} \mathrm{N}$, Calandra T, et al. An essential regulatory role for macrophage migration inhibitory factor in T-cell activation. Proc Natl Acad Sci U S A. 1996, 93: 7849-54.

24. Bernhagen Jürgen, Krohn Regina, Lue Hongqi et al. MIF is a noncognate ligand of CXC chemokine receptors in inflammatory and atherogenic cell recruitment. Nat Med, 2007, 13: 587-96.

25. Lang Tali, Lee Jacinta P W, Elgass Kirstin, et al. Macrophage migration inhibitory factor is required for NLRP3 inflammasome activation. Nat Commun, 2018, 9: 2223.

26. Kim Hee Kyung, Garcia Alvaro Baeza, Siu Edwin, et al. Macrophage migration inhibitory factor regulates innate $\gamma \delta$ T-cell responses IL-17 expression. FASEB J, 2019, 33, 6919-6932.

27. Mizue Y, Ghani S, Leng L, et al. Role for Macrophage Migration Inhibitory Factor (MIF) in Asthma. Proc Natl Acad Sci USA. 2005; 102:14410-14415.

28. Connelly K L, Kandane-Rathnayake R, Hoi A et al. Association of MIF, but not type I interferon-induced chemokines, with increased disease activity in Asian patients with systemic lupus erythematosus. Sci Rep, 2016, 6: 29909.

29. Foote A, Briganti EM, Kipen Y, et al. Macrophage migration inhibitory factor in Systemic Lupus Erythematosus. J Rheumatol. 2004; 31:268-273.

30. Lan H Y, Yang N, Nikolic-Paterson D J, et al. Expression of macrophage migration inhibitory factor in human glomerulonephritis. Kidney Int, 2000, 57, 499-509. 
31. De la Cruz-Mosso U, Bucala R, Palafox-Sanchez CA, et al. Macrophage migration inhibitory factor: association of -794 CATT5-8 and $-173 \mathrm{G}>\mathrm{C}$ polymorphisms with TNF-alpha in systemic lupus erythematosus. Hum Immunol, 2014, 75:433-9.

32. Sánchez E, Gómez LM, Lopez-Nevot MA, et al. Evidence of association of macrophage migration inhibitory factor gene polymorphisms with systemic lupus erythematosus. Genes Immun, 2006, 7:433-6. doi:10.1038/sj.gene.6364310

33. Sreih A, Ezzeddine R, Leng L, et al. Dual effect of the macrophage migration inhibitory factor gene on the development and severity of human systemic lupus erythematosus. Arthritis Rheum, 2011, 63:3942-51. doi:10.1002/art.30624

34. Kerjaschki Dontscho, Pathomechanisms and molecular basis of membranous glomerulopathy. Lancet, 2004, 364: 1194-6.

35. Sasaki Satoshi, Nishihira Jun, Ishibashi Teruo et al. Transgene of MIF induces podocyte injury and progressive mesangial sclerosis in the mouse kidney. Kidney Int., 2004, 65: 469-81.

36. Boswell JM, Yui MA, Endres S, et al. Novel and enhanced IL-1 gene expression in autoimmune mice with lupus. J Immunol (1988) 141:118-24.

37. Lemay S, Mao C, Singh AK. Cytokine gene expression in the MRL/Ipr model of lupus nephritis. Kidney Int (1996) 50:85-93. doi:10.1038/ki.1996.290

38. McCarthy EM, Smith S, Lee RZ, et al. The association of cytokines with disease activity and damage scores in systemic lupus erythematosus patients. Rheumatology (2014) 53:1586-94. doi:10.1093/rheumatology/ket428

39. Umare V, Pradhan V, Nadkar M, et al. Effect of proinflammatory cytokines (IL-6, TNFa, and IL-1 $\beta$ ) on clinical manifestations in Indian SLE patients. Mediators Inflamm (2014) 2014:8.doi:10.1155/2014/385297

40. Kitani A, Hara M, Hirose T, et al. Autostimulatory effects of IL-6 on excessive B cell differentiation in patients with systemic lupus erythematosus: analysis of IL-6 production and IL-6R expression. Clin Exp Immunol. 1992;88 (1):75-83.

41. Kitani A, Hara M, Hirose T, et al. Heterogeneity of B cell responsiveness to interleukin 4, interleukin 6 and low molecular weight $B$ cell growth factor in discrete stages of B cell activation in patients with systemic lupus erythematosus. Clin Exp Immunol.1989;77 (1):31-36.

42. Tilg $H$, Trehu $E$, Atkins MB, et al. Interleukin-6 (IL-6) as an anti-inflammatory cytokine: induction of circulating IL-1 receptor antagonist and soluble tumor necrosis factor receptor p55. Blood. 1994;83 (1):113-118.

43. Randolph, G. J, M. B. Furie. A soluble gradient of endogenous monocyte chem. oattractant protein-1 promotes the transendothelial migration of monocytes in vitro. J Immunol. 1995, 155: 3610-3618.

44. Kim Hyun Lee, Lee Dong-Sup, Yang Seung Hee, et al. The polymorphism of monocyte chemoattractant protein-1 is associated with the renal disease of SLE. Am J Kidney Dis. 2002, 40, 1146-52. 
45. Lin J, Zhou Z, Huo R, etc. Cyr61 induces IL-6 production by fibroblast-like synoviocytes promoting Th17 differentiation in rheumatoid arthritis. J Immunol. 2012 Jun 1;188 (11):5776-84. doi: 10.4049/jimmunol.1103201.

46. Zhai T, Gao C, Huo R, etc. Cyr61 participates in the pathogenesis of rheumatoid arthritis via promoting MMP-3 expression by fibroblast-like synoviocytes. Mod Rheumatol. 2017 May;27 (3):466475. doi: 10.1080/14397595.2016.1220447.

47. You JJ, Yang $\mathrm{CH}$, Yang $\mathrm{CM}$, Chen MS. Cyr61 induces the expression of monocyte chemoattractant protein-1 via the integrin av $\beta 3, \mathrm{FAK}, \mathrm{PI} 3 \mathrm{~K} / \mathrm{Akt}$, and NF-KB pathways in retinal vascular endothelial cells. Cell Signal. 2014 Jan;26 (1):133-40. doi: 10.1016/j.cellsig.2013.08.026.

48. Lin J, Li N, Chen H, etc. Serum Cyr61 is associated with clinical disease activity and inflammation in patients with systemic lupus erythematosus. Medicine (Baltimore). 2015 May;94 (19):e834. doi: 10.1097/MD.0000000000000834.

\section{Figures}
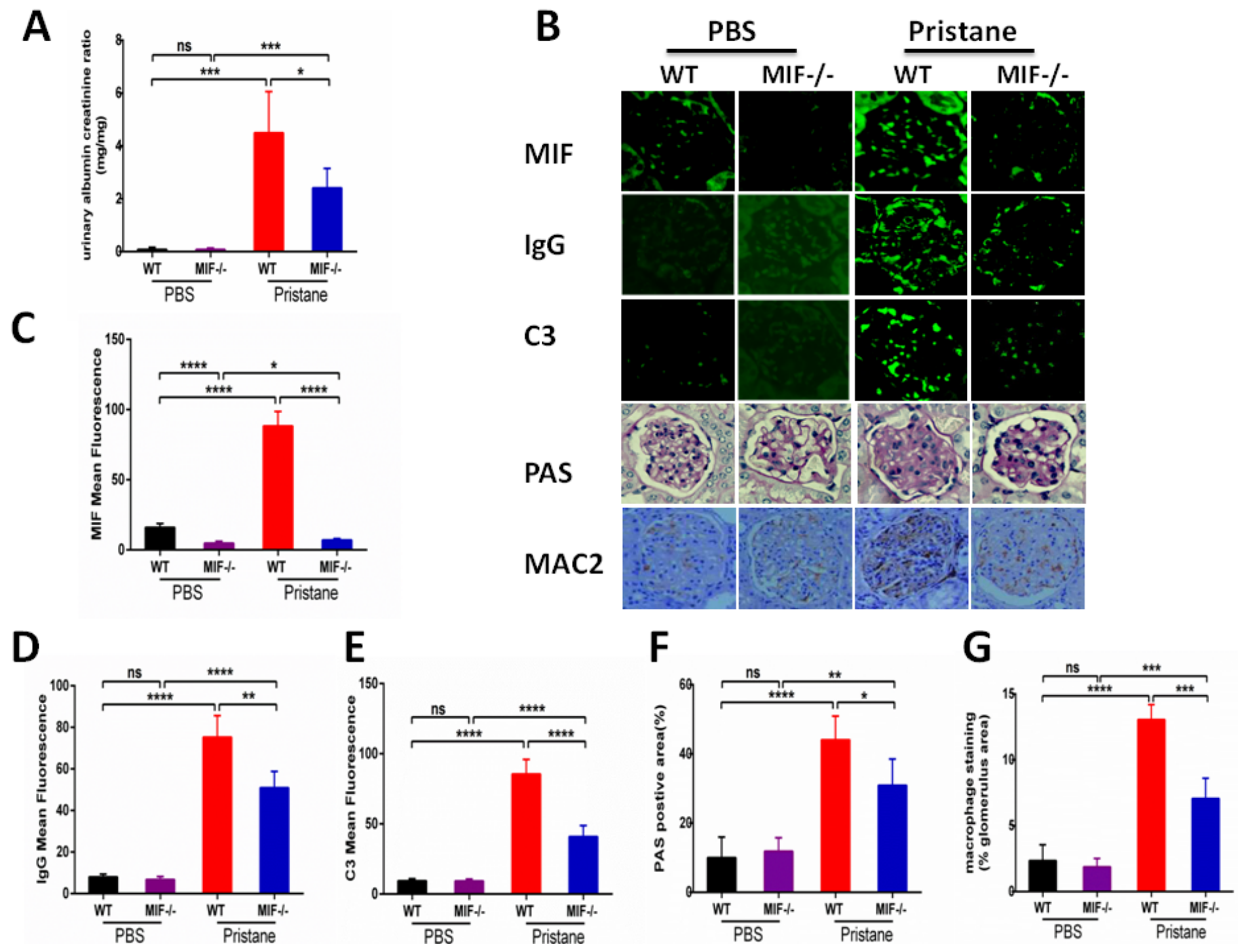
Figure 1

Urinary albumin excretion, kidney histopathology and macrophage infiltration in lupus-prone mice induced by pristane. (A) The urinary albumin/creatinine ratio was significantly decreased in the Mif-/pristane group when compared with the pristane group (P凶0.05). (B) and (C), (D) and (E) MIF, ICs and C3 deposition were significantly increased in mice treated with pristane, and there were less ICs and C3 deposition in Mif-/- mice. (B) and (F)The PAS-positive area in C57BL/6 mice treated with pristane is significantly larger than Mif-/- mice than in wild-type mice. (B) and (G) There were less macrophages infiltration in Mif-/- mice treated with pristane. Figure A, C, D, E, F and G were analyzed by Mann-Whitney

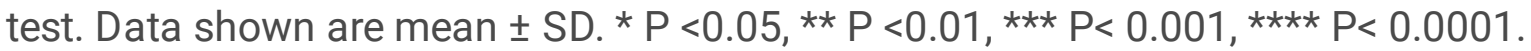

A

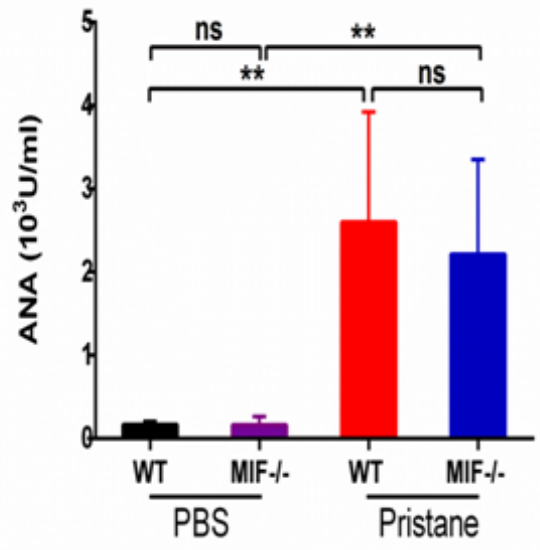

D

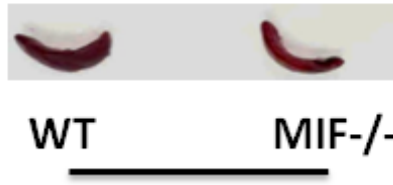

PBS
B

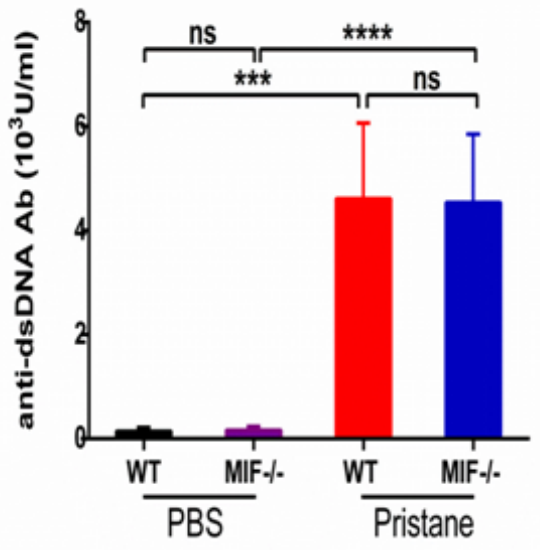

E

\section{C}
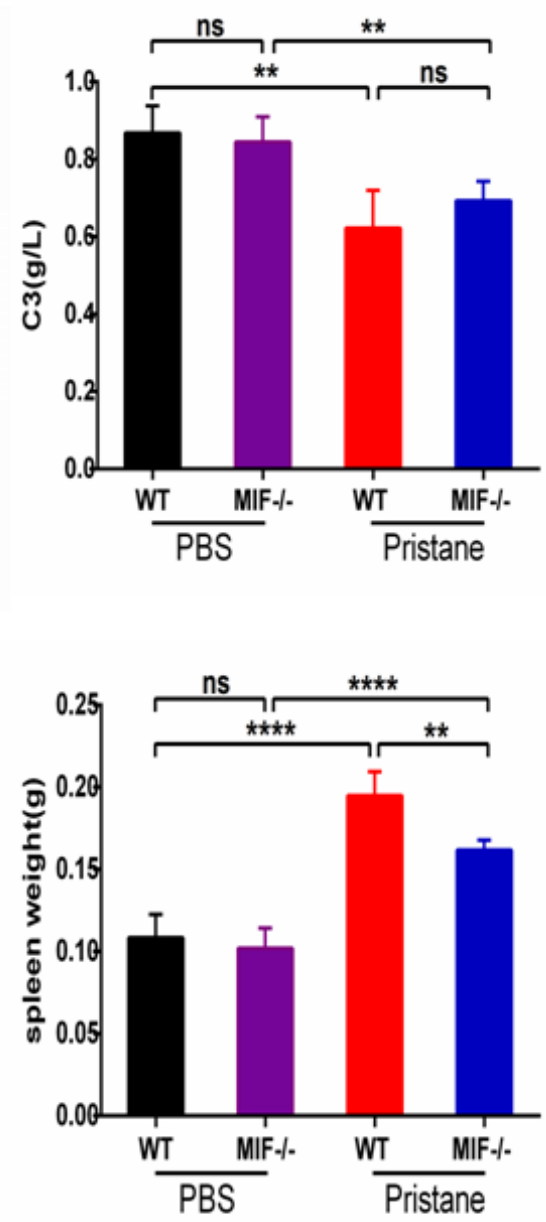

Figure 2

Sera ANA, anti-dsDNA and complement C3 expression in mice with pristane-induced lupus. Spleens were smaller in Mif-/- mice after pristane treatment. (A), (B) and (C) The expression of ANA and anti-dsDNA was significantly increased in mice treated with pristane (P区0.01) and the expression of C3 was significantly decreased in mice treated with pristane $(P \bowtie 0.01)$, while there were no significant differences 
between the Mif-/- and wild type C57BL/6 mice after pristane induction of SLE. (D) and (E) Both the volume and weight were significantly decreased in Mif-/- mice treated with pristane when compared to the corresponding wild-type mice. Figure $A, B, C$ and $E$ were analyzed by Mann-Whitney test. Data shown are mean $\pm S D$. * $P<0.05$, ** $P<0.01$, *** $P<0.001$, **** $P<0.0001$.

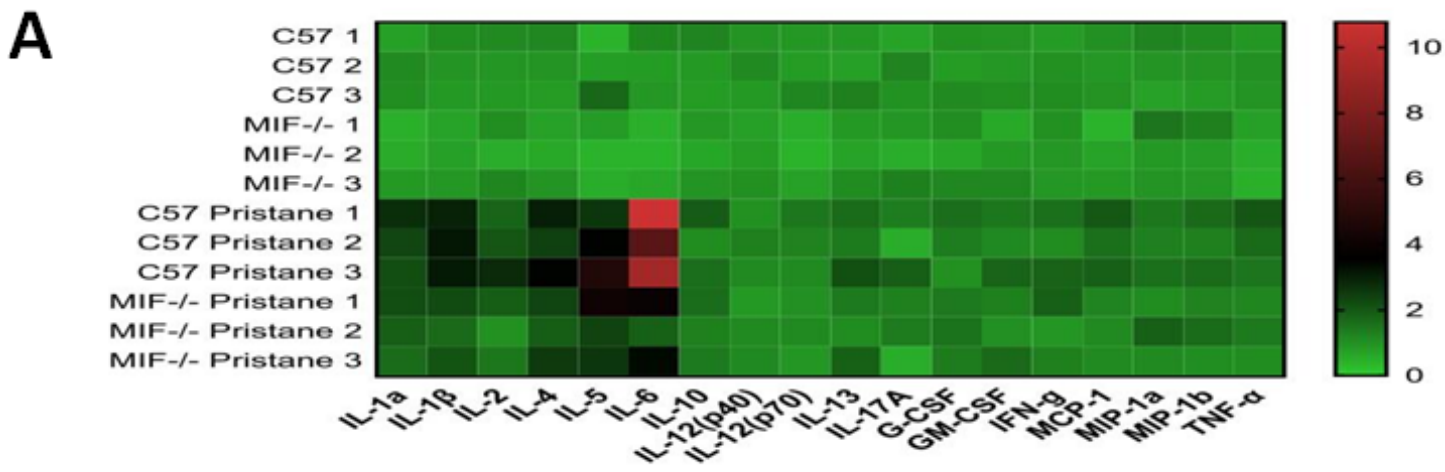

B

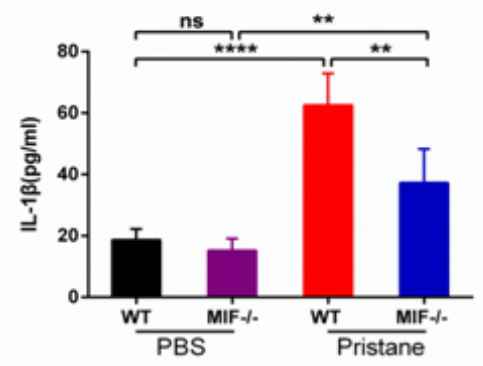

E

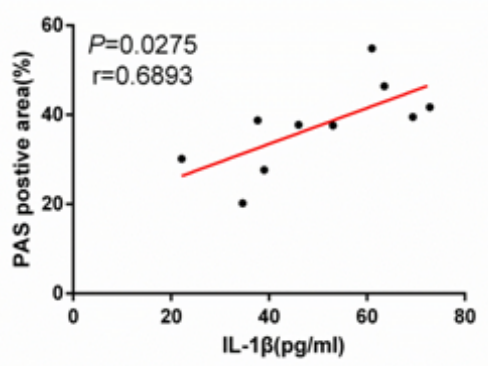

C

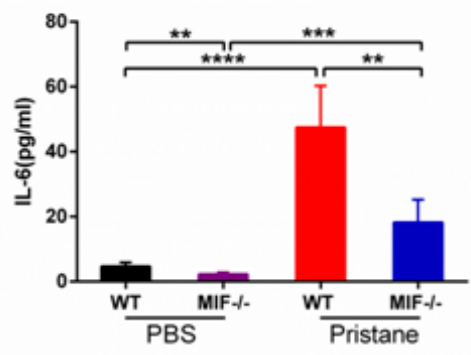

$\mathbf{F}$

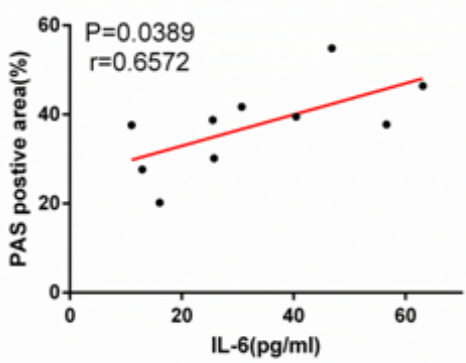

D

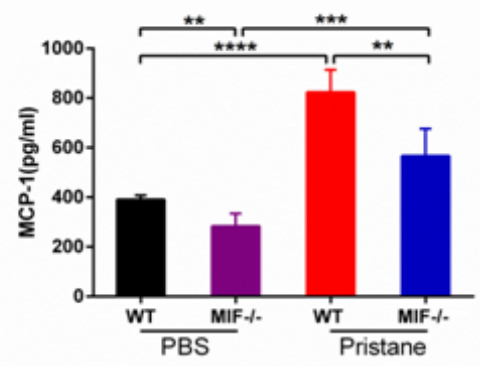

\section{G}

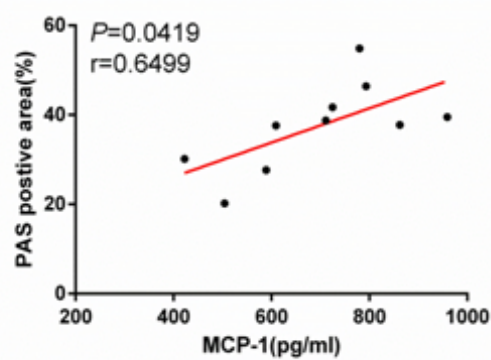

\section{Figure 3}

Mif-/- mice have reduced inflammatory cytokine expression when compared to wild type mice. (A) The expression of 18 sera cytokines including IL-1a, IL-1 $\beta$, IL-6, MCP-1, TNF-a, IL-2, IL-4, IL-5, IL-10, IL-12 (p40), IL-12 (p70), IL-13, IL-17A, G-CSF, GM-CSF, IFN-g, MIP-1 and MIP-1. (B), (C) and (D) IL-1 $\beta$, IL-6 and MCP-1 expression were detected by ELISA. After treatment with pristane, the expression of IL-1 $\beta$, IL-6 and MCP-1 were significantly increased, and there were less IL-1 $\beta$, IL- 6 and MCP-1 expression in the pristine-treated Mif-/- group when compared with the pristine-treated wild-type group. (D), (E) and (F) IL-1 3 , IL-6, and MCP1 expression is positively associated with PAS-positive areas in kidney. Figure $B, C$ and $D$ were analyzed by Mann-Whitney test. Figure E, F and G were analyzed by nonparametric Spearman's correlation. Data shown are mean $\pm S D$. * $P<0.05$, ** $P<0.01$, *** $P<0.001$, **** $P<0.0001$. 
A

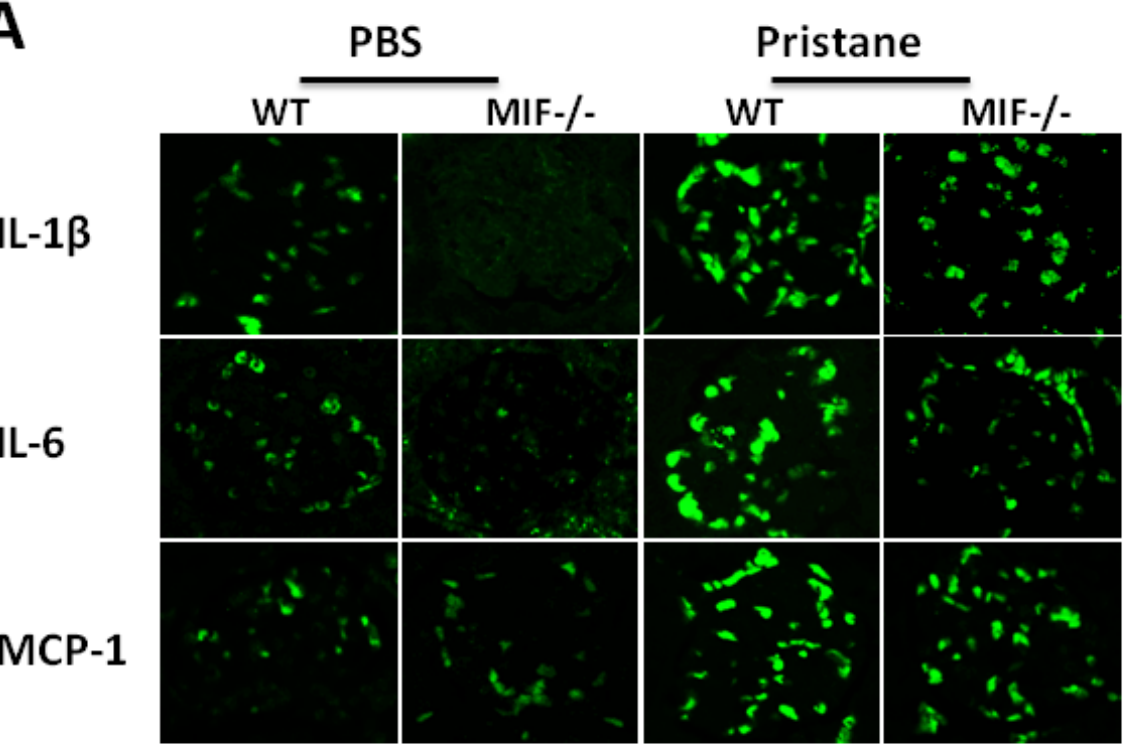

B
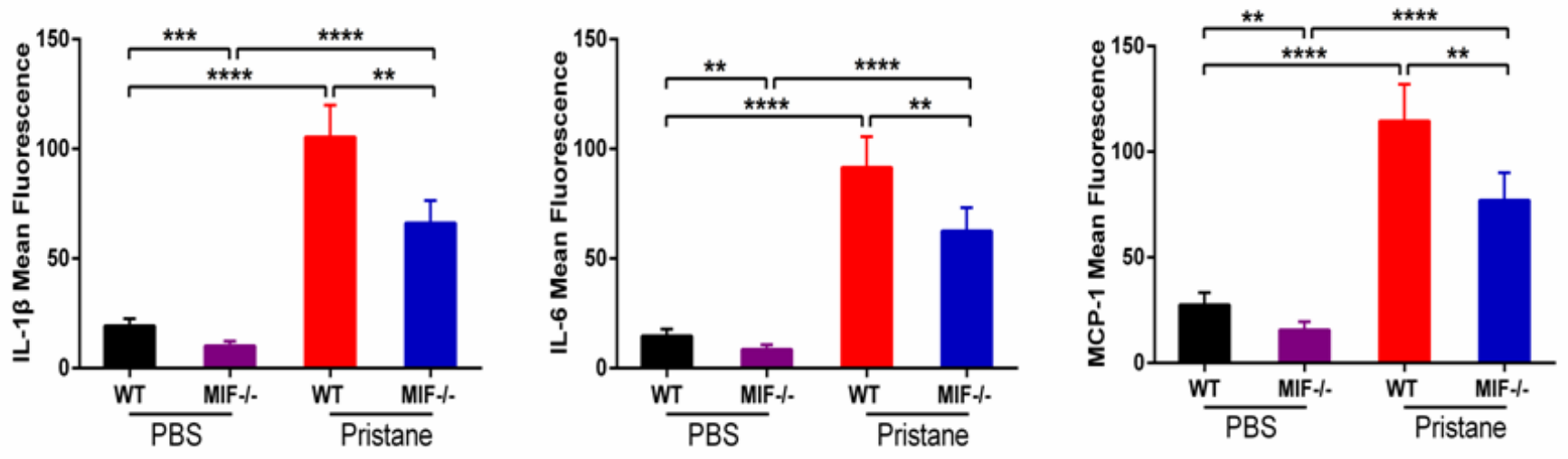

Figure 4

MIF promotes the expression of IL-1 $\beta$, IL-6 and MCP-1 in glomeruli. (A) The expression of IL-1 $\beta$, IL- 6 and MCP-1 in renal glomeruli. After treatment with pristane, the expression of IL-1 $\beta$, IL-6 and MCP-1 was significantly increased, while there were less expression of IL-1 $\beta$, IL-6 and MCP-1 in Mif-/-than in wild-type mice. (B), (C), and (D) IL-1 $\beta$, IL-6 and MCP-1 expression were significantly decreased in BMDM from Mif-/mice. MIF promotes the expression of IL-1 $\beta$, IL-6 and MCP-1 in BMDMs. Figure B, C and D were analyzed by Mann-Whitney test. Data were shown as mean $\pm S D$. ** $P<0.01$, *** $P<0.001, * \star \star \star P<0.0001$. 
A

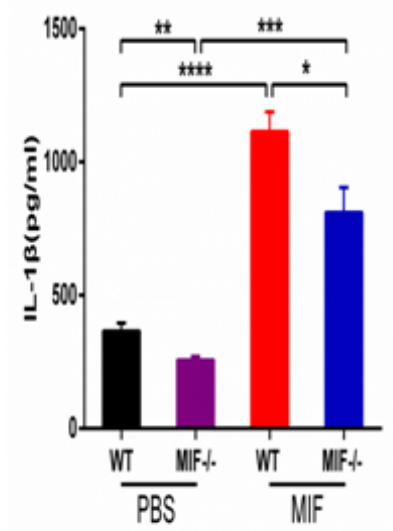

E

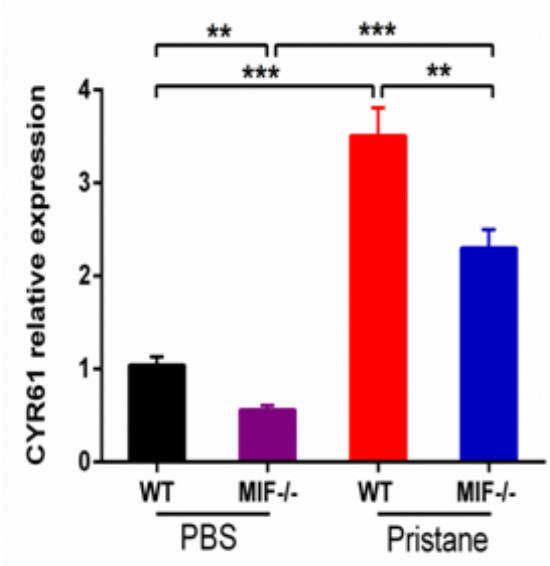

B

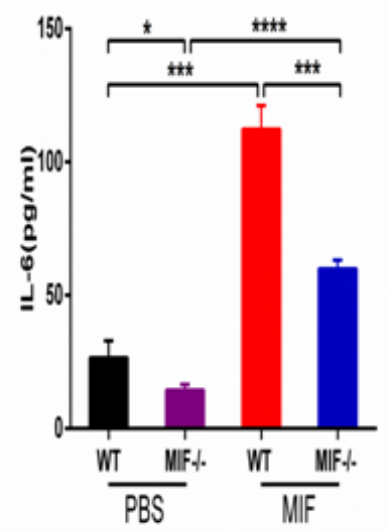

$\mathbf{F}$
C

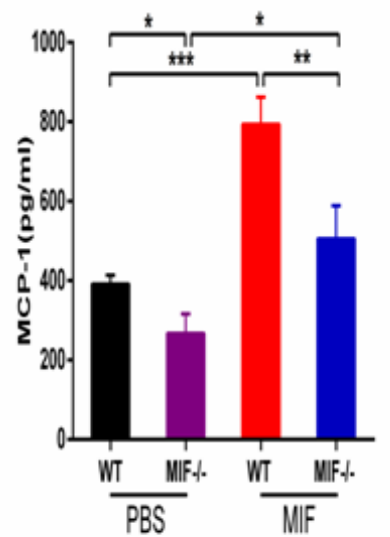

D

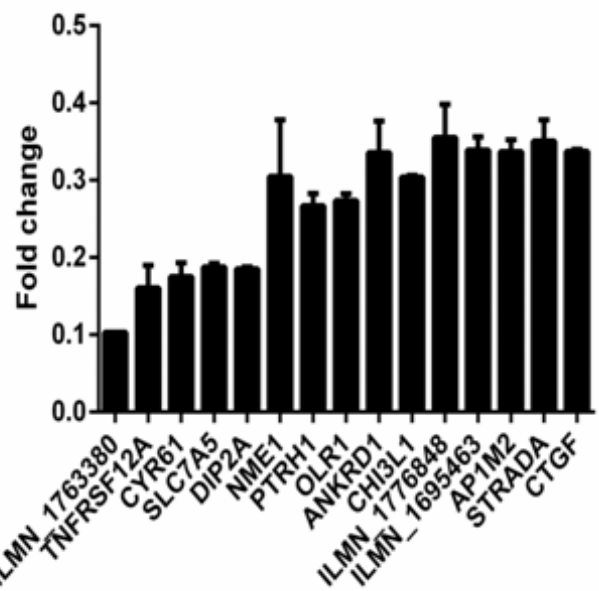

G
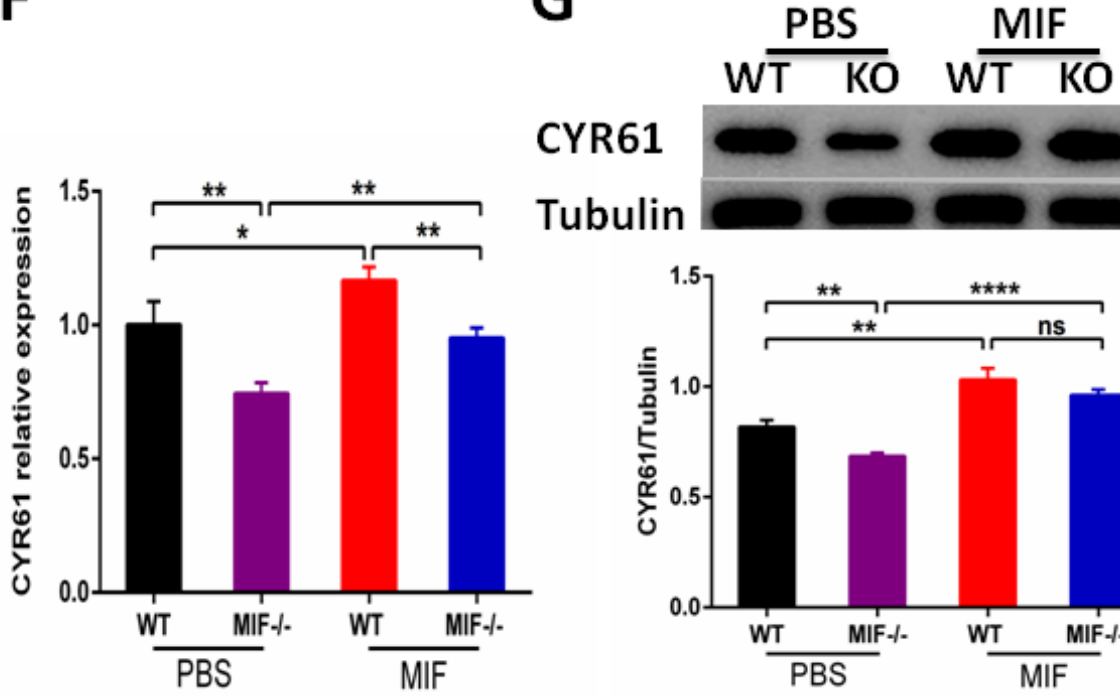

CYR61

Tubulin

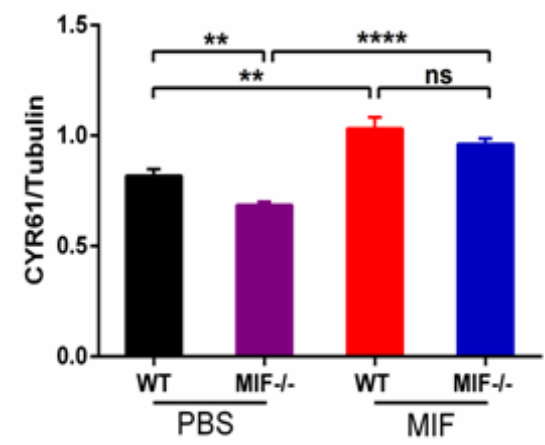

Figure 5

MIF promotes the expression of IL-1 $1 \beta$, IL- 6 and MCP-1 and CYR61 in BMDM. (A), (B) and (C) The expression of IL-1 $\beta$, IL- 6 and MCP-1 were reduced in Mif-/- BMDMs, and MIF can promote the expression of IL-1 $\beta$, IL-6 and MCP-1. (D) Among the 15 lowest genes in Mif siRNA knockdown HEK293cells, CYR61, ANKRD1 and CTGF are key molecules involved in hippo signaling. (E) The expression of CYR61 mRNA was significantly reduced in the kidneys from Mif-/-mouse. The expression of CYR61 was significantly increased in the kidneys from pristine-induced lupus mouse. (F) The expression of CYR61 mRNA was significantly reduced in the Mif-/- BMDMs compared with WT BMDMs. The expression of CYR61 was significantly increased by stimulating with MIF. (G) The expression of CYR61 protein was significantly reduced in the Mif-/-BMDMs compared with WT BMDMs. The expression of CYR61 protein was significantly increased by stimulating with MIF in BMDMs. Figure A, B, C, E, F and G were analyzed by

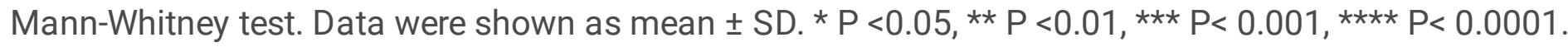


A

\section{NC CYR61}

\section{CYR61}

Tubulin

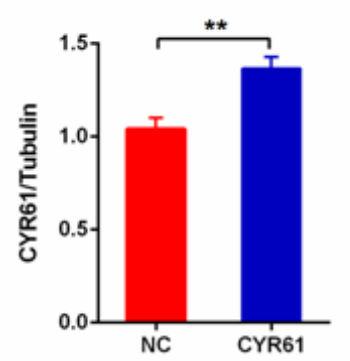

E

\section{NC siCYR61}

CYR61

Tubulin

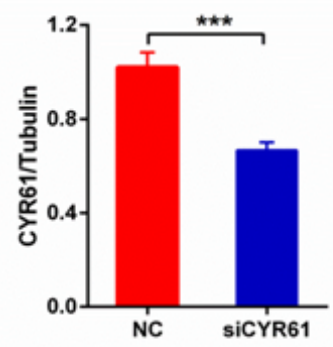

B

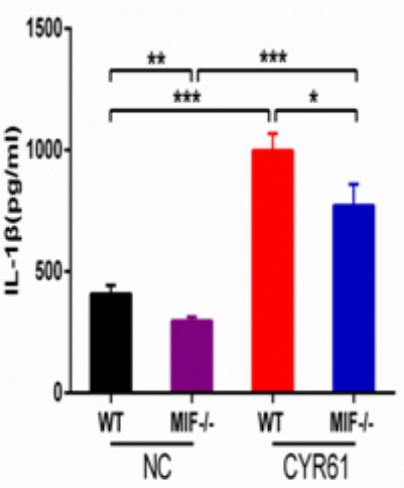

F

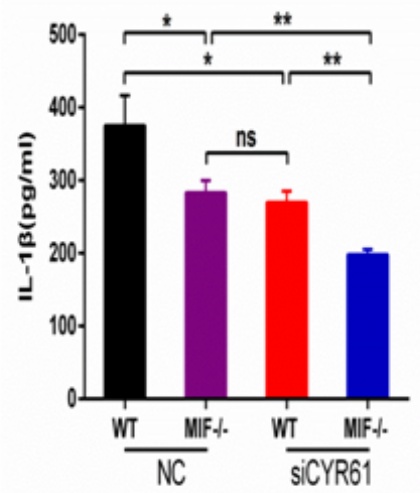

C

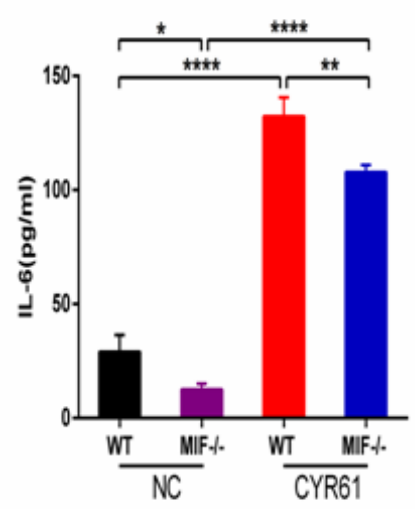

G

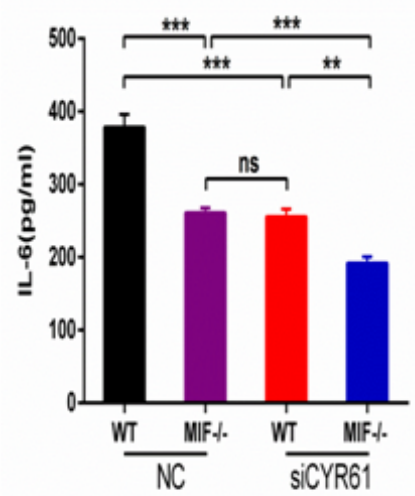

D

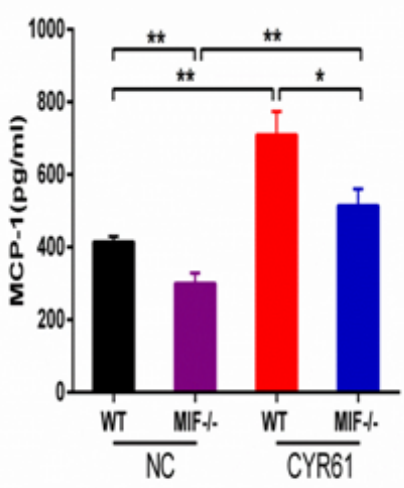

H

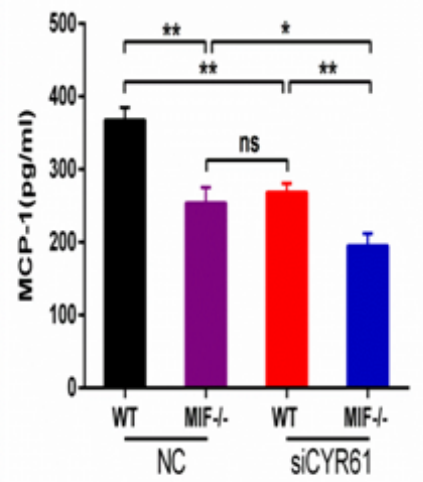

Figure 6

MIF promotes the expression of IL-1 $\beta$, IL- 6 and MCP-1 via CYR61 in BMDM. (A) The expression of CYR61 protein was significantly increased in BMDMs after transfected with pCMV6-CYR61 vector. (B), (C) and (D) The expression of IL-1 $\beta$, IL-6 and MCP-1 were increased in BMDMs transfected with pCMV6-CYR61 vector. (E) The expression of CYR61 protein was significantly decreased in BMDMs after transfected with CYR61 siRNA. (F), (G) and (H) The expression of IL-1 $\beta$, IL- 6 and MCP-1 were decreased in BMDMs transfected with CYR61 siRNA. Figure $A, B, C, D, E, F$ and $G$ were analyzed by Mann-Whitney test. Data

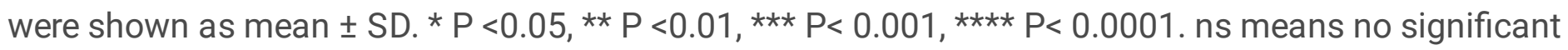
difference. 